\title{
Producción y uso de artefactos líticos en contextos cazadores-recolectores del Área Interserrana (Argentina): análisis integral de la diversidad tecno-morfológica y funcional
}

\author{
Agustina MassigoGe \\ CONICET - INCUAPA (Universidad Nacional del Centro de la Provincia de Buenos Aires) \\ amassigo@soc.unicen.edu.ar \\ Nélida PAL \\ CONICET - Centro Austral de Investigaciones Científicas - INCUAPA \\ nelidapal@yahoo.com.ar
}

Recibido: 23 de octubre de 2009

Aceptado: 23 de junio de 2010

\begin{abstract}
RESUMEN
En este trabajo se integran los resultados de los análisis tecno-morfológico y funcional de base microscópica de los instrumentos líticos de los sitios Cortaderas y Las Brusquillas 1, localizados en el Área Interserrana (región pampeana, Argentina), correspondientes a ocupaciones cazadoras-recolectoras del Holoceno tardío inicial (3.500 - 2.000 años A.P.). Los objetivos de este trabajo son contribuir a la caracterización funcional de los contextos arqueológicos bajo estudio, aportar al conocimiento de las estrategias tecnológicas involucradas en la producción y uso de los instrumentos líticos en la micro-región de estudio y en el Área Interserrana y evaluar la correspondencia entre los resultados de los análisis tecno-morfológico y funcional.
\end{abstract}

Palabras clave: Análisis tecno-morfológico, análisis funcional, cazadores-recolectores, Holoceno tardío, Área Interserrana (Argentina), tecnología lítica.

\section{Production and Use of Lithic Artifacts in Hunter-Gatherer Contexts in the Interserrana Area (Argentina): Integrative Analysis of Techno-Morphological and Functional Diversity}

\begin{abstract}
In this paper the results of techno-morphological and functional analyses of lithic tools from the sites Cortaderas and Las Brusquillas 1, located in the Interserrana Area (Pampean region, Argentina) and corresponding to early Late Holocene hunter-gatherers (3.500-2.000 B.P.), are presented. The aim of this paper is to contribute to the definition of the sites functions, and to the understanding of the technological strategies employed in tool production and use by hunter-gatherers in the Interserrana Area. Additionally, this paper aims to evaluate the correlation between the techno-morphological and functional data.
\end{abstract}

Key words: Techno-morphological analysis, functional analysis, hunter-gatherers, Late Holocene, Interserrana Area (Argentina), lithic technology.

SUMARIO: 1. Introducción. 2. Características generales de la micro-región y de los sitios arqueológicos bajo estudio. 3. Metodología. 4. Resultados del sitio Cortaderas. 5. Resultados del sitio Las Brusquillas 1. 6. Discusión. 7. Conclusiones. 8. Referencias bibliográficas.

\section{Introducción}

En los últimos años se ha comenzado a desarrollar de manera complementaria el análisis tecno-morfológico y funcional de base microscópica de los conjuntos líticos en diversos sitios de la región pampeana (Argentina). La finalidad de estas investiga- 
ciones es explicar los procesos técnicos desarrollados en los sitios e identificar las actividades en las cuales los artefactos líticos habrían participado como herramientas de trabajo (Castro de Aguilar 1987-1988; Flegenheimer y Leipus 2007; Landini et. al. 2000; Leipus 2006; Pal 2009a; Sacur Silvestre 2004).

Actualmente se reconoce que la conjunción de ambas líneas metodológicas permite llevar a cabo un estudio más completo de la tecnología lítica, posibilitando el acercamiento a diferentes esferas de las sociedades cazadoras-recolectoras, tales como el aprovechamiento de los recursos líticos, los patrones de subsistencia y la movilidad (Álvarez 2003; Leipus 2006). La tecnología es entendida, en este caso, como un fenómeno complejo que involucra factores ambientales y sociales dado que envuelve, por un lado, el conocimiento y las habilidades técnicas para confeccionar un producto $\mathrm{y}$, por otro, las acciones físicas por parte de los actores sociales que toman sus decisiones influenciados por el entorno social y ambiental (Álvarez 2003).

En este trabajo se integran los resultados obtenidos del análisis tecno-morfológico y de huellas de uso de los conjuntos líticos de los sitios Cortaderas y Las Brusquillas 1 (Área Interserrana, región pampeana; Figura 1), correspondientes a ocupaciones cazadoras-recolectoras del Holoceno tardío inicial (3.500 a 2.000 años A.P.). Los objetivos de este trabajo son: 1) contribuir a la caracterización funcional de los contextos arqueológicos bajo estudio, combinando la información tecno-morfológica y funcional de los conjuntos líticos con los resultados surgidos del análisis de otras líneas de evidencia, 2) incrementar el conocimiento de las estrategias tecnológicas involucradas en la producción y uso de los instrumentos líticos implementadas por las sociedades cazadoras-recolectoras del Holoceno tardío en la micro-región de estudio y en el Área Interserrana, y 3) evaluar la correspondencia entre los resultados de ambos tipos de análisis del material lítico.

El análisis funcional de base microscópica contribuye a la identificación de los materiales y sustancias trabajadas y los modos de uso de los instrumentos líticos. Así, posibilita conocer parte de las actividades desarrolladas en los sitios, específicamente aquellas vinculadas a la explotación de distintos recursos, sobre todo los que no se preservan en el registro arqueológico (p.e., madera, piel). Asimismo, este tipo de estudio permite explorar la influencia que pudo tener el contexto de uso sobre el diseño y la organización de la tecnología.

Los conjuntos instrumentales recuperados en los sitios Cortaderas y Las Brusquillas 1 están integrados, en términos generales, por los mismos grupos tipológicos (raederas, raspadores, etc.). Asimismo, ambos conjuntos comparten una serie de características tecnológicas, incluyendo la selección preferencial del mismo tipo de materia prima, el uso de lascas internas, mayormente medianas y espesas, como formas base para la manufactura de los instrumentos, lascados preferencialmente en situación unifacial directa y dominio del retoque marginal. En relación con los aspectos indicados, resulta de interés determinar, mediante la realización del análisis funcional de base microscópica, si las similitudes tipológicas y tecnológicas observadas obedecen a semejanzas en los procesos productivos desarrollados en estos yacimientos. Coincidiendo con Álvarez (2004), se piensa que la relación entre la variación tecno-morfológica y funcional ha de ser plasmada en forma de hipótesis que deben ser contrastadas con los datos obtenidos del estudio de las huellas de uso. 


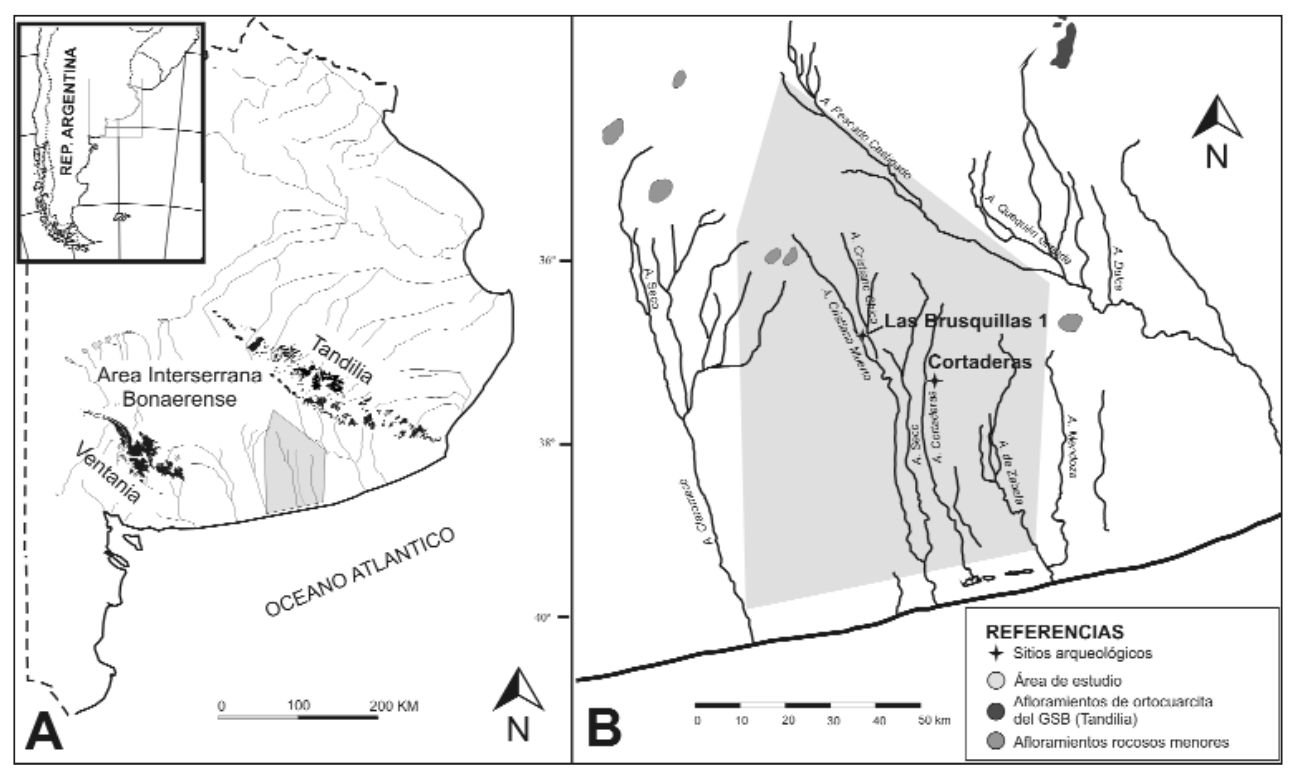

Figura 1: $\mathrm{A}=$ localización de la micro-región de estudio dentro del Área Interserrana; $\mathrm{B}=$ ubicación de los sitios analizados en este trabajo

\section{Características generales de la micro-región y de los sitios arqueológicos bajo estudio}

Los sitios considerados en este estudio se localizan en una micro-región ubicada en el sector centro-meridional del Área Interserrana (ver Figura 1), que está siendo investigada desde el año 2004 por una de las autoras (Massigoge 2007, 2009). El Área Interserrana comprende la llanura que se extiende entre los sistemas serranos de Ventania y Tandilia, la costa Atlántica y la depresión de Carhué, dentro de la denominada sub-región Pampa Húmeda (Politis 1986). En esta llanura, el paisaje es el de una planicie con leves ondulaciones, dominada por morfologías eólicas y con cuencas fluviales de desarrollo incipiente (Zárate y Rabassa 2005). Dentro del área de estudio no se encuentran cursos de agua de gran desarrollo, sólo pequeños arroyos de carácter permanente que presentan muy pocos afluentes.

En el Área Interserrana las rocas aptas para la talla son escasas y su distribución se restringe al litoral atlántico donde se presentan en forma de depósitos de cantos rodados de pequeñas dimensiones, y a afloramientos rocosos pequeños de toba silicificada y metacuarcita en el interior de la llanura (Bonomo 2005; Flegenheimer y Bayón 2002). No obstante, en las sierras de Ventania y Tandilia la oferta de recursos líticos es muy importante y variada, principalmente en el segundo cordón (Flegenheimer y Bayón 2002).

El sitio Cortaderas (CO; $\left.38^{\circ} 19^{\prime} 15^{\prime \prime} \mathrm{S}, 59^{\circ} 39^{\prime} 47^{\prime \prime} \mathrm{W}\right)$ se localiza sobre la margen izquierda del arroyo homónimo (ver Figura 1). Las investigaciones en este sitio se iniciaron en la década de 1980 (Politis et al. 1983) y fueron retomadas en el 2004 (Massi- 
goge 2007). Una parte significativa del conjunto lítico obtenido en las excavaciones tempranas se ha extraviado, por lo que los resultados presentados en este trabajo corresponden a la muestra recuperada en las excavaciones realizadas recientemente $\left(16 \mathrm{~m}^{2}\right)$.

Las evidencias que integran la unidad arqueológica denominada Componente Inferior (CI) fueron recuperadas en un depósito sedimentario de origen fluvio-lacustre, sobre el cual se desarrolló un suelo de carácter cumúlico. Un fechado ${ }^{14} \mathrm{C}$ por AMS arrojó una edad de $2.270 \pm 190$ años A.P. (AA-67736; óseo), ubicando las ocupaciones en momentos tempranos del Holoceno tardío. El CI está compuesto por una asociación de artefactos líticos y cerámicos, fragmentos de pigmento mineral y especímenes faunísticos correspondientes a distintos taxa (Massigoge 2007).

Los estudios tecno-morfológicos del material lítico permiten proponer que en el sitio se desarrollaron gran parte de las actividades de la secuencia de reducción lítica, encontrándose mejor representadas las tareas finales de manufactura de los instrumentos. Por otra parte, los análisis faunísticos indican que en el sitio se procesaron y consumieron distintos recursos animales, principalmente guanaco (Lama guanicoe). Las distintas líneas de evidencia sugieren que el sitio CO habría funcionado como un campamento residencial (Massigoge 2007, 2009).

El sitio Las Brusquillas 1 (LB1; 38 16 $21^{\prime \prime} \mathrm{S}, 59^{\circ} 47^{\prime} 35^{\prime \prime} \mathrm{W}$ ), se emplaza sobre una lomada baja, a unos $200 \mathrm{~m}$ de distancia de la margen derecha del arroyo Cristiano Chico (ver Figura 1) y fue excavado en el año $2006\left(14 \mathrm{~m}^{2}\right)$. La secuencia estratigráfica corresponde a un depósito eólico en cuya sección cuspidal se desarrolló el suelo actual. Aunque no se registraron discordancias sedimentarias y los materiales arqueológicos se encontraban distribuidos de modo continuo en sentido vertical, el conjunto se segregó en dos unidades arqueológicas denominadas Niveles Superiores (NS) y Componente Inferior (CI), sobre la base de las diferencias en las clases de materiales recuperados en las partes superior e inferior del depósito (Massigoge 2009).

Los NS se caracterizan por la presencia de materiales históricos, tiestos cerámicos, fragmentos de pigmento mineral, artefactos líticos y especímenes óseos. Este conjunto es el resultado de la mezcla de materiales de ocupaciones prehistóricas y post-conquista. Por otra parte, el CI se caracteriza por la presencia de artefactos líticos, fragmentos de pigmento mineral y restos faunísticos correspondientes principalmente a guanaco. Un fechado ${ }^{14} \mathrm{C}$ por AMS efectuado sobre un espécimen de este taxón procedente del CI, dio una edad de $3.334 \pm 43$ A.P. (AA-81453; diente), ubicando las ocupaciones en momentos iniciales del Holoceno tardío (Massigoge 2009).

Los materiales líticos de ambas unidades arqueológicas se tratarán en conjunto en este trabajo por dos razones. Primero, los conjuntos líticos de las dos unidades muestran similitudes en la representación porcentual de las distintas propiedades tecnomorfológicas analizadas (p.e., materias primas, tamaños y módulos dimensionales de desechos e instrumentos, tipos de lascas, etc.). Segundo, el estudio de los procesos de formación del sitio señala que la bioturbación produjo la movilización vertical de los materiales. Teniendo en cuenta que el pico máximo de la distribución vertical de los materiales líticos se registró en los niveles arqueológicos asociados al CI, se infirió que el depósito del conjunto lítico, o al menos una parte significativa de éste, se produjo durante las ocupaciones prehistóricas representadas en esta última unidad arqueológica (Massigoge 2009). 
El análisis tecno-morfológico indica que durante las ocupaciones prehistóricas se desarrollaron actividades correspondientes a distintas etapas de la secuencia de reducción lítica, encontrándose mejor representadas las últimas etapas de la manufactura de artefactos líticos. Por otra parte, el estudio faunístico muestra que los ocupantes del sitio habrían llevado a cabo actividades de procesamiento y consumo de recursos faunísticos, principalmente guanaco y de modo complementario ñandú (Rhea americana). En términos generales, las actividades realizadas en este sitio coinciden con las inferidas para CO. No obstante, la explotación casi exclusiva de guanaco y el amplio predominio de los elementos apendiculares sobre los axiales, sugirieron que las actividades desarrolladas en LB1 fueron de carácter más específico que en CO. Los estudios llevados a cabo hasta el momento no han permitido precisar la funcionalidad del CI de LB1 (Massigoge 2009).

\section{Metodología}

Para el estudio tecno-morfológico de los instrumentos se siguieron los criterios planteados por Aschero $(1975,1983)$, mientras que para el análisis funcional de base microscópica se aplicaron las propuestas desarrolladas por Semenov (1964), Keeley (1980) y Mansur (1986/1990, 1999), entre otros. La identificación de las huellas de uso se realizó empleando un microscopio metalográfico Olympus BHM con un rango de aumento desde 100X a 500X, priorizándose los 200X. Para el análisis funcional se seleccionaron todos los instrumentos enteros y aquéllos fragmentados que conservan como mínimo un filo de $2 \mathrm{~cm}$ de longitud con una morfología rectilínea y levemente convexa.

\section{Resultados del sitio Cortaderas}

\subsection{Análisis tecno-morfológico}

El conjunto lítico del CI está integrado por instrumentos $(\mathrm{n}=27 ; 10,7 \%)$ y desechos de talla $(n=226 ; 89,3 \%)$. Si bien entre los materiales recuperados en el año 2004 no se registran núcleos, esta clase de objetos está presente entre los materiales procedentes de la colección de 1980, aunque en muy baja frecuencia $(n=2)$. En cuanto a los instrumentos $(\mathrm{n}=27)$, la mayoría fueron manufacturados por medio de lascados, y sólo uno fue confeccionado mediante la técnica de picado y abrasión. Con respecto a las materias primas, predomina ampliamente la ortocuarcita Grupo Sierras Bayas (GSB) y sólo se registra un instrumento de ftanita y uno de una roca granítica; asimismo, no se observa presencia de corteza en ninguno de ellos. Por otra parte, es bajo el número de instrumentos enteros $(\mathrm{n}=6)$; la mayoría están fracturados no significativamente (cuando se conserva más del $90 \%$ del instrumento original) o corresponden a fragmentos de instrumentos (Massigoge 2007, 2009).

Considerando el conjunto de instrumentos por talla, se observa que el grupo tipológico mayoritario es el de las raederas $(\mathrm{n}=8)$, seguido por el de los instrumentos com- 
puestos $(\mathrm{n}=4)$, raspadores y filos en bisel asimétrico ( $\mathrm{n}=1$ cada uno); el resto de los artefactos tallados son fragmentos no diferenciados de artefactos. Entre los artefactos compuestos se registra un filo en raspador combinado con dos filos en raedera, un filo en raedera combinado con un filo de retoque sumario y dos filos en raedera combinados con perforador.

Algunos aspectos destacados del conjunto de instrumentos son: (1) alta frecuencia de filos dobles; (2) selección de lascas internas como formas base; (3) predominio de módulo de tamaño mediano grande, del módulo de longitud-anchura mediano normal y del módulo de anchura-espesor espeso; (4) lascados mayoritariamente en situación unifacial directa y (5) preponderancia del retoque marginal (Massigoge 2007, 2009).

\subsection{Análisis funcional de base microscópica}

El análisis funcional se realizó sobre un total de 21 instrumentos que presentan un total de 40 filos retocados (Cuadro 1). En este conjunto predominan las piezas con filos dobles $(n=14)$, seguidas por las simples $(n=6)$ y triples $(n=1)$; asimismo, se registran tres puntas destacadas que se analizaron de modo independiente. El análisis funcional permite sugerir un buen estado de conservación de los instrumentos; en este sentido, en la totalidad de las piezas se registró un leve lustre de suelo y sólo en un $15 \%$ de los filos $(n=6)$ se identificó una fuerte alteración que impidió su identificación funcional. Considerando los filos en los cuales la alteración no impidió la inferencia funcional, se observa que el 70,6\% $(n=24)$ exhibe huellas de utilización y el 29,4\% $(n=10)$ no fue utilizado. El buen desarrollo de las huellas de uso en algunos de los filos podría vincularse con un tiempo prolongado de utilización de los artefactos.

En cuanto a los materiales procesados, los filos fueron utilizados principalmente sobre hueso $(20,8 \%$; $n=5)$, materiales duros no determinables, tales como hueso o madera $(20,8 \% ; n=5)$, piel $(12,5 \% ; n=3)$, madera $(8,3 \% ; n=2)$ y materiales blandos de origen animal, entre los que se podrían incluir carne, tendones y piel $(8,3 \% ; n=2)$. En algunos casos, debido al escaso desarrollo de las huellas de uso, no se pudo identificar ni la dureza relativa ni el material trabajado $(29,2 \% ; n=7)$. En relación con la cinemática de trabajo, el $66,7 \%(\mathrm{n}=16)$ de los filos fue usado en acciones longitudinales (p.e., corte, aserrado), el 20,8\% ( $\mathrm{n}=5)$ en actividades transversales (p.e., raspado, alisado, desbaste) y el $4,2 \%(n=1)$ en movimientos rotatorios (p.e., perforación); mientras que en el $8,3 \%(\mathrm{n}=2)$ de los filos no se logró identificar el movimiento ejecutado.

No se ha registrado ningún filo que muestre la superposición de huellas producidas por el trabajo sobre distintos materiales o por el empleo de diferentes cinemáticas. Por otra parte, en un $66,7 \%(\mathrm{n}=10)$ de los artefactos con filos dobles o triples se registran huellas de uso en más de un filo (47,6\% del total de instrumentos). Entre estos se observa el uso de distintos filos y/o puntas tanto para trabajar el mismo material (p.e., madera) como para trabajar materiales distintos (p.e., hueso y material blando de origen animal). Asimismo, los diferentes filos se emplearon tanto con la misma cinemática como con movimientos diferentes (ver Cuadro 1).

Tal como se muestra en la Figura 2, las tareas que involucraron el empleo de instrumentos en $\mathrm{CO}$ corresponden mayoritariamente al aserrado de materiales duros, entre ellos hueso $(20,8 \%$; Figura 3$)$, madera $(8,3 \%)$ y materiales duros no determina- 
A. Massigoge y N. Pal Producción y uso de artefactos líticos en contextos cazadores-recolectores...

Cuadro 1: Propiedades tecno-morfológicas y funcionales de los instrumentos de Cortaderas

\begin{tabular}{|c|c|c|c|c|c|c|c|c|c|c|c|}
\hline \multirow[b]{2}{*}{$\begin{array}{l}\text { Número } \\
\text { de pieza }\end{array}$} & \multicolumn{4}{|c|}{ Atributos de la pieza } & \multicolumn{4}{|c|}{ Atributos de los filos } & \multicolumn{3}{|c|}{ Análisis funcional } \\
\hline & 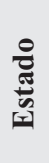 & & 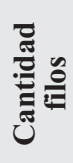 & 递告 & 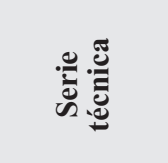 & 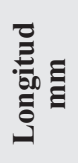 & $\underset{\substack{0 \\
0}}{0}$ & 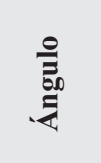 & $\stackrel{\ominus}{\emptyset}$ & 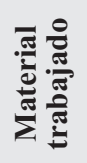 & 氖 \\
\hline \multirow{2}{*}{ FCS.CO.515 } & \multirow{2}{*}{$\mathrm{F}$} & \multirow{2}{*}{$\mathrm{C}$} & \multirow{2}{*}{2} & \multirow{2}{*}{ FND } & MR & NA & NA & NA & NDA & & \\
\hline & & & & & MR & NA & NA & NA & NDA & & \\
\hline \multirow{2}{*}{ FCS.CO.517 } & \multirow{2}{*}{$\mathrm{F}$} & \multirow{2}{*}{$\mathrm{C}$} & \multirow{2}{*}{2} & \multirow{2}{*}{ FND } & MR & NA & NA & $25^{\circ}$ & US & $\mathrm{H}$ & $\mathrm{L}$ \\
\hline & & & & & MR & NA & NA & $42^{\circ}$ & UP & $\mathrm{I}$ & $\mathrm{L}$ \\
\hline \multirow{2}{*}{ FCS.CO.521 } & \multirow{2}{*}{$\mathrm{F}$} & \multirow{2}{*}{$\mathrm{C}$} & \multirow{2}{*}{2} & \multirow{2}{*}{ RAE } & RQ-M & 39,9 & $\mathrm{CV}$ & $65^{\circ}$ & UP & $\mathrm{I}$ & $\mathrm{L}$ \\
\hline & & & & & RQ-M & 41 & $\mathrm{CV}$ & $65^{\circ}$ & US & MD & $\mathrm{L}$ \\
\hline FCS.CO.523 & $\mathrm{F}$ & $\mathrm{C}$ & 1 & FND & RQ-M & NA & $\mathrm{CV}$ & $50^{\circ}-75^{\circ}$ & NU & & \\
\hline FCS.CO.525 & $\mathrm{F}$ & $\mathrm{C}$ & 1 & RAE (frag) & RQ-M & 39,3 & $\mathrm{CV}$ & $60^{\circ}$ & US & $\mathrm{H}$ & $\mathrm{L}$ \\
\hline \multirow{2}{*}{ FCS.CO.531 } & \multirow{2}{*}{$\mathrm{F}$} & \multirow{2}{*}{$\mathrm{C}$} & & & RQ-M & NA & NA & $50^{\circ}$ & NU & & \\
\hline & & & 2 & FND & RQ-PE & NA & NA & $50^{\circ}$ & NU & & \\
\hline FCS.CO.536 & $\mathrm{F}$ & $\mathrm{C}$ & 1 & FND & RQ-M & NA & $\mathrm{CV}$ & $80^{\circ}$ & NU & & \\
\hline & & & & COMP & RQ-M & 47 & $\mathrm{CV}$ & $55^{\circ}$ & US & I & $\mathrm{L}$ \\
\hline FCS.CO.538 & $\mathrm{E}$ & $\mathrm{C}$ & 3 & (RAS+ & RQ-PE & 47 & $\mathrm{CV}$ & $55^{\circ}$ & US & I & $\mathrm{L}$ \\
\hline & & & & RAE) & RQ-M & 25,7 & $\mathrm{CV}$ & $75^{\circ}$ & US & $\mathrm{P}$ & $\mathrm{T}$ \\
\hline FCS.CO.542 & $\mathrm{F}$ & FT & 1 & RAS & RQ-M & NA & $\mathrm{CV}$ & $75^{\circ}$ & NU & & \\
\hline FCS.CO.550 & $\mathrm{F}$ & $\mathrm{C}$ & 1 & FND & RQ-M & nd & NA & $40^{\circ}$ & NU & & \\
\hline FCS CO.556 & $\mathrm{F}$ & $\mathrm{C}$ & & COMP & RQ/RT-PE & NA & $\mathrm{CV}$ & $60^{\circ}$ & UP & I & $\mathrm{T}$ \\
\hline FCS.CU.J56 & $\mathrm{F}$ & C & 2 & (RAE+FRS) & RQ-M & NA & $\mathrm{CC}$ & $74^{\circ}$ & UP & I & $\mathrm{L}$ \\
\hline $\mathrm{ECS} C O 557$ & $\mathrm{~F}_{\mathrm{O}}$ & $C$ & 2 & $P A F$ & RQ-PE & 70 & $\mathrm{CV}$ & $70^{\circ}$ & UP & MD & I \\
\hline FEs. CU.ग & $\mathrm{E}$ & C & 2 & RAE & RQ-M & 35 & $\mathrm{CV}$ & $51^{\circ}$ & UP & I & I \\
\hline & & & & & RQ/RT-PE & 42,5 & $\mathrm{CV}$ & $58^{\circ}$ & US & $\mathrm{H}$ & $\mathrm{L}$ \\
\hline FCS.CO.561 & $\mathrm{E}$ & $\mathrm{C}$ & $2+1$ & $\begin{array}{l}\text { COMP } \\
(\mathrm{RAE}+\mathrm{PE})\end{array}$ & RQ/RT-PE & 52 & $\mathrm{CV}$ & $45^{\circ}$ & US & MBA & $\mathrm{T}$ \\
\hline & & & & & PUN & A DES & $\mathrm{CAD}$ & & $\mathrm{NU}$ & & \\
\hline FCS CO 562 & $\mathrm{~F}$ & $\mathrm{C}$ & 2 & FBA & MR & NA & $\mathrm{CV}$ & $50^{\circ}$ & NDA & & \\
\hline & & & & & MR & NA & $\mathrm{R}$ & $55^{\circ}$ & NDA & & \\
\hline & & & & & RQ-M/RT-PE & 66,4 & $\mathrm{CV}$ & $50^{\circ}$ & US & MD & $\mathrm{L}$ \\
\hline FCS.CO.565 & $\mathrm{E}$ & $\mathrm{C}$ & $2+1$ & $\begin{array}{l}\text { COMP } \\
(\mathrm{RAE}+\mathrm{PE})\end{array}$ & MR & 66,4 & $\mathrm{CV}$ & $35^{\circ}$ & US & $\mathrm{H}$ & $\mathrm{L}$ \\
\hline & & & & & PUN & TA DES & $\mathrm{ACAD}$ & & NU & & \\
\hline FCS CO 569 & $\mathrm{~F}$ & $\mathrm{C}$ & 2 & $\mathrm{RAF}$ & MR/RQ-M & NA & $\mathrm{CV}$ & $40^{\circ}$ & US & MD & $\mathrm{L}$ \\
\hline & & & & & RQ/RT-PE & NA & $\mathrm{CV}$ & $70^{\circ}$ & US & $\mathrm{H}$ & $\mathrm{L}$ \\
\hline FCS CO 592 & $\mathrm{~F}$ & $\mathrm{C}$ & 2 & RAE & RQ/RT-PE & NA & $\mathrm{R}$ & $60^{\circ}$ & NDA & & \\
\hline FCS.CU.592 & $\mathrm{F}$ & C & 2 & (frag) & RQ/RT-PE & NA & $\mathrm{R}$ & $65^{\circ}$ & NU & & \\
\hline FCS $C O 593$ & $\mathrm{~F}$ & $\mathrm{C}$ & 2 & $\mathrm{RAF}$ & MR/RQ-M & 40,1 & $\mathrm{R}$ & $50^{\circ}$ & NDA & & \\
\hline FCS. CU.593 & $\mathrm{F}$ & C & 2 & KAE & MR/RQ-M & 43,4 & $\mathrm{CV}$ & $50^{\circ}$ & UP & MD & $\mathrm{L}$ \\
\hline & & & & & RQ/RT-E & 53,5 & $\mathrm{CV}$ & $60^{\circ}$ & US & $\mathrm{P}$ & $\mathrm{T}$ \\
\hline FCS.CO.594 & $\mathrm{E}$ & $\mathrm{C}$ & $2+1$ & RAE & RQ/RT-E & 53,5 & $\mathrm{CV}$ & $60^{\circ}$ & US & MBA & $\mathrm{T}$ \\
\hline & & & & & PUN & A DES & $\mathrm{CAD}$ & & US & $\mathrm{P}$ & $\mathrm{R}$ \\
\hline FCS CO 599 & $\mathrm{~F}$ & $\mathrm{C}$ & 2 & RAF & MR/RQ-M & 57 & $\mathrm{CV}$ & $55^{\circ}$ & US & M & $\mathrm{L}$ \\
\hline FCS.CU.599 & $\mathrm{E}$ & C & 2 & KAE & MR/RQ-M & 57 & $\mathrm{CV}$ & $55^{\circ}$ & US & M & $\mathrm{L}$ \\
\hline FCS.CO.664 & $\mathrm{F}$ & $\mathrm{C}$ & 1 & FND & MR & NA & NA & $45^{\circ}$ & NU & & \\
\hline
\end{tabular}

Clave: $\mathrm{F}=$ fracturado; $\mathrm{E}=$ entero; $\mathrm{C}=$ ortocuarcita; $\mathrm{FT}=\mathrm{ftanita} ; \mathrm{FND}=$ fragmento no diferenciado de instrumento; $\mathrm{RAE}=$ raedera $; \mathrm{FRAG}=$ fragmento $\mathrm{COMP}=$ artefacto compuesto $; \mathrm{RAS}=$ raspador; $\mathrm{FRS}=$ filo con retoque $\mathrm{su}-$ mario; $\mathrm{PE}=$ perforador; $\mathrm{MR}=$ microretoque; $\mathrm{RQ}=$ retoque; $\mathrm{RQ}-\mathrm{M}=$ retoque marginal; $\mathrm{RQ}-\mathrm{PE}=$ retoque parcialmente extendido; RT-PE = retalla parcialmente extendida; RT-E = retalla extendida; NA= no aplica por fractura; $\mathrm{CV}=$ convexo; $\mathrm{CC}=$ cóncavo; $\mathrm{R}=$ recto; $\mathrm{NDA}=$ no determinado por alteración; $\mathrm{US}=$ uso seguro; $\mathrm{UP}=$ uso posible; $\mathrm{NU}=$ no utilizado; $\mathrm{H}=$ hueso; $\mathrm{I}=$ indeterminado; $\mathrm{M}=$ madera; $\mathrm{MD}=$ material duro; $\mathrm{P}=$ piel; $\mathrm{MBA}=$ material blando de origen animal; $\mathrm{L}=$ longitudinal; $\mathrm{T}=$ transversal; $\mathrm{R}=$ rotatorio 

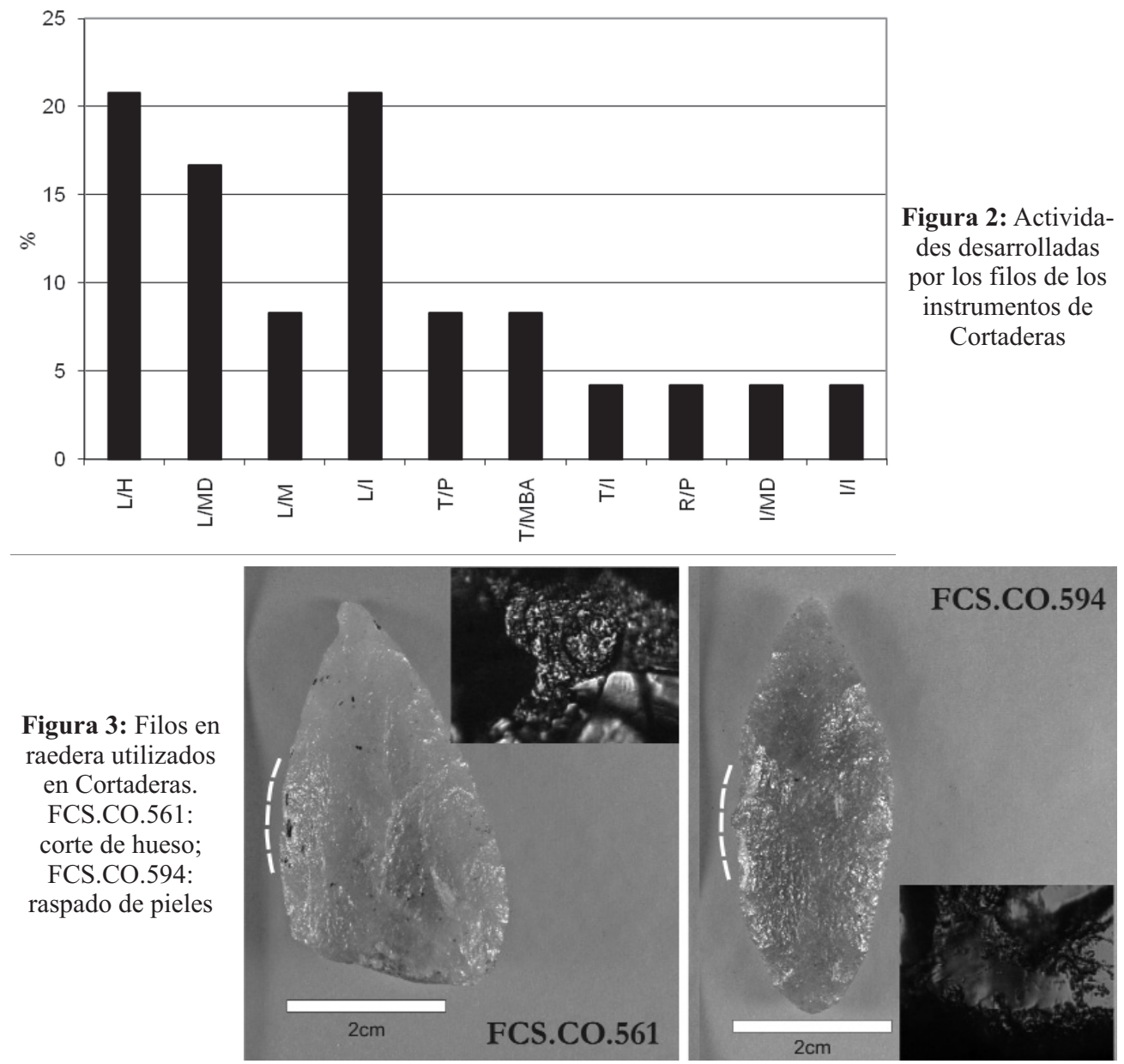

dos (16,7\%); y en menor proporción al raspado ( $8,3 \%$; ver Figura 3 ) y perforado de pieles $(4,2 \%)$ y al raspado de materiales blandos de origen animal que también podrían corresponder al procesamiento de pieles $(8,3 \%)$. Dado que las huellas de uso correspondientes a hueso se encuentran poco desarrolladas y aisladas, se plantea que los artefactos participaron en tareas en las cuales los instrumentos sobrellevan un contacto esporádico con el material óseo, por ejemplo el procesamiento de animales. Por otra parte, el trabajo sobre pieles indica el procesamiento intensivo de pieles frescas; de modo complementario, la identificación de estrías oscuras en los biseles sugiere el empleo de algún tipo de abrasivo en estas actividades.

\subsection{Integración de la información tecno-morfológica y funcional}

La totalidad de los instrumentos que presentan evidencias de utilización se encuentran manufacturados sobre ortocuarcita y el único instrumento de ftanita no ha sido utilizado. Con respecto a la clasificación tecno-morfológica de los filos con huellas 
de uso, se observan 19 filos en ra.edera, 1 filo en raspador, 1 filo con retoque sumario, 2 fragmentos de filos de instrumentos no diferenciados y 1 punta destacada.

Con respecto a la relación entre categorías tecno-morfológicas, material trabajado y cinemática (Cuadro 2), se determinó que los filos en raedera fueron usados para cortar/aserrar hueso, madera y materiales duros no determinados, raspar piel y materiales blandos de origen animal y procesar materiales no identificados con movimientos longitudinales y transversales. Por su parte, el filo con retoque sumario se empleó para trabajar un material no determinado con movimiento longitudinal, el filo en raspador se utilizó para raspar pieles y la punta destacada se empleó para perforar este mismo material. Por último, los fragmentos de artefactos evidencian trabajo longitudinal sobre hueso y sobre una sustancia no determinada.

La comparación de la información funcional y tecno-morfológica no permite sostener que los filos hayan sido seleccionados de acuerdo con su forma y características técnicas para el desarrollo de actividades específicas. En cuanto al primer atributo, debe mencionarse que la gran mayoría de los filos estudiados son convexos (ver Cuadro 1), sugiriendo que esta forma de filo se utilizó para el desempeño de una gran diversidad de funciones. Con respecto al segundo atributo, si bien se registra una gran variabilidad en las series técnicas entre los distintos filos, ésta no se vincula con los

Cuadro 2: Materiales trabajados y actividades desarrolladas por tipo de filo en los dos sitios analizados

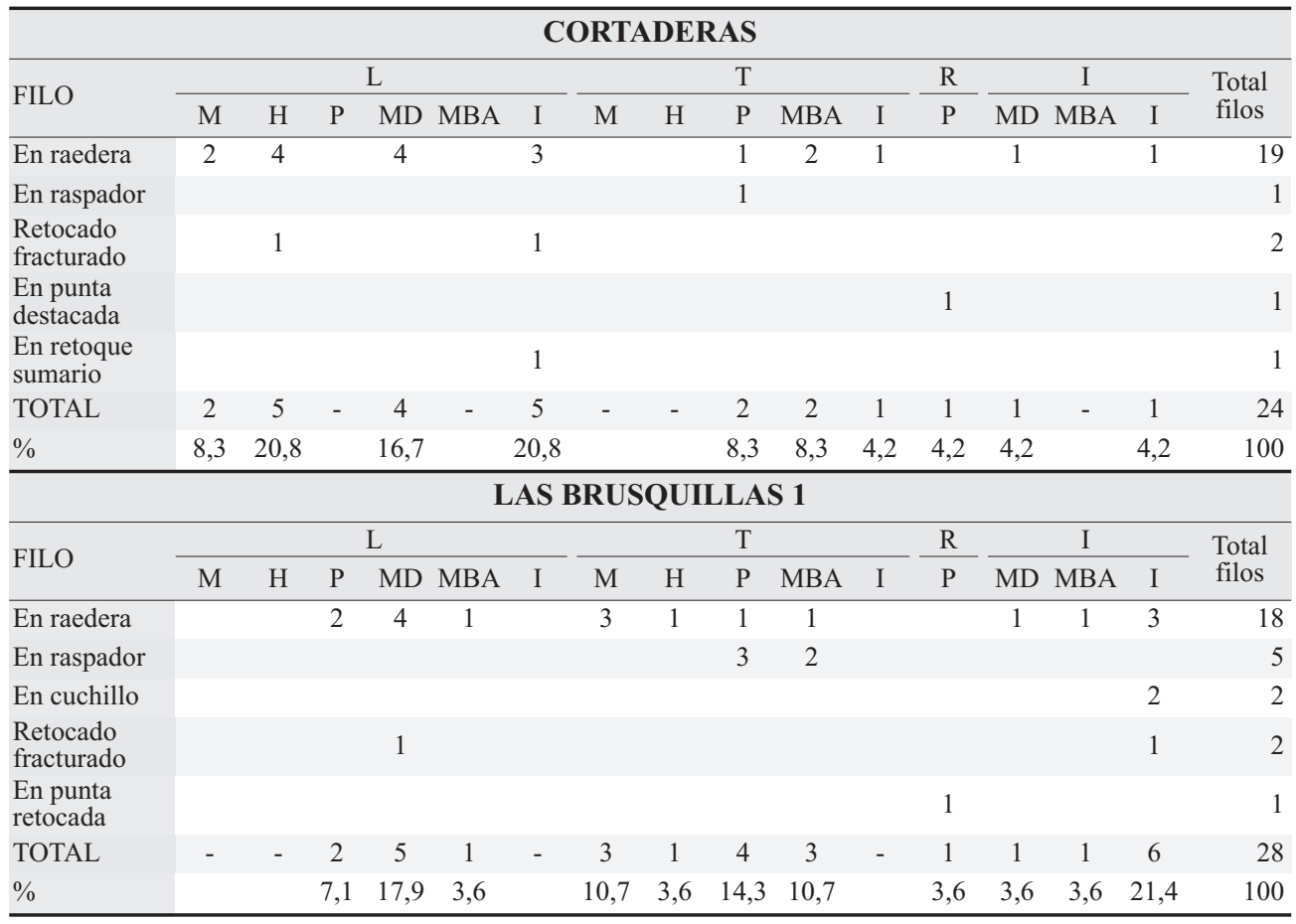

Clave: $\mathrm{L}=$ longitudinal; $\mathrm{T}=$ transversal; $\mathrm{R}=$ rotatorio; $\mathrm{I}=$ indeterminado; $\mathrm{M}=$ madera; $\mathrm{H}=$ hueso; $\mathrm{P}=$ piel; $\mathrm{MD}=$ material duro; $\mathrm{MBA}=$ material blando de origen animal 
diferentes usos a los que fueron sometidos (ver Cuadro 1). Por otro lado, se observan diferencias entre la longitud y el ángulo del bisel y las actividades desarrolladas por los filos. En cuanto a la primera variable, el promedio de los filos utilizados es de 49,2 $\mathrm{mm}$ y las mayores diferencias se producen en la media de los filos que rasparon cuero $(39,6 \mathrm{~mm})$ y aserraron sobre madera $(57 \mathrm{~mm})$. En cuanto a los ángulos, la totalidad se incluye dentro de la categoría agudos. Sin embargo también se observa una distinción entre los que rasparon cuero y los que aserraron madera, cuyas medias son $67,5^{\circ}$ y $49,6^{\circ}$, respectivamente.

\section{Resultados del sitio Las Brusquillas 1}

\subsection{Análisis tecno-morfológico}

En LB1 se recuperaron instrumentos $(n=34 ; 3,1 \%)$, desechos de talla $(n=1071$; $96,4 \%)$ y núcleos $(\mathrm{n}=5 ; 0,5 \%)$. Todos los instrumentos fueron confeccionados por talla, utilizando principalmente ortocuarcita GSB $(70,6 \%)$ y, secundariamente, otras rocas como ftanita $(20,6 \%)$, dolomía silicificada $(5,9 \%)$ y una roca silícea no identificada $(2,9 \%)$. Ninguno de los instrumentos presenta corteza. Por otra parte, sólo unos pocos se encuentran enteros $(n=12)$; la mayoría están fragmentados de manera no significativa o corresponden a fragmentos de instrumentos.

En cuanto a los grupos tipológicos, se observa que los tipos más frecuentes son las raederas $(\mathrm{n}=8)$ y los raspadores $(\mathrm{n}=7)$. Otros grupos representados por menor número de piezas son los artefactos compuestos $(\mathrm{n}=3)$, las muescas retocadas, los cuchillos de filo retocado y los de filo bisel asimétrico ( $\mathrm{n}=1$ cada uno). Asimismo, gran número de ítems corresponden a fragmentos no diferenciados de instrumentos $(\mathrm{n}=13)$. En el grupo de los artefactos compuestos, se observa la combinación de filos en raspador con filos en raedera y con filo de retoque sumario.

En el conjunto de los instrumentos se observan las siguientes tendencias tecnomorfológicas: (1) predominio de más de un filo por artefacto; (2) utilización de lascas internas como formas base; (3) mayor frecuencia de módulos de tamaño medianos y módulos de anchura-espesor muy espeso y espeso, y diversidad de módulos de longitud-anchura; (4) lascados preferencialmente en situación unifacial directa y (5) dominio del retoque marginal (Massigoge 2009).

\subsection{Análisis funcional de base microscópica}

El análisis funcional se realizó sobre un total de 45 filos correspondientes a 25 instrumentos (Cuadro 3). En este conjunto predominan las piezas con filos dobles $(\mathrm{n}=11)$, seguidas por las de filos simples $(\mathrm{n}=10)$ y triples $(\mathrm{n}=4)$; además, en uno de los artefactos se registró una punta destacada con huellas de uso.

A través del estudio microscópico se determinó que la mayoría de las piezas presentan un leve lustre de suelo. Asimismo, un 26,7\% de los filos ( $\mathrm{n}=12)$ muestran severas alteraciones posteriores al depósito producto de procesos físico-químicos que intervinieron en la identificación funcional. Gran número de los filos alterados $(n=10)$ 
A. Massigoge y N. Pal Producción y uso de artefactos líticos en contextos cazadores-recolectores...

Cuadro 3: Propiedades tecno-morfológicas y funcionales de los instrumentos de Las Brusquillas 1

\begin{tabular}{|c|c|c|c|c|c|c|c|c|c|c|c|}
\hline \multirow[b]{2}{*}{$\begin{array}{c}\text { Número de } \\
\text { pieza }\end{array}$} & \multicolumn{4}{|c|}{ Atributos de la pieza } & \multicolumn{4}{|c|}{ Atributos de los filos } & \multicolumn{3}{|c|}{ Análisis funcional } \\
\hline & 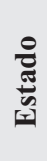 & 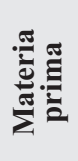 & 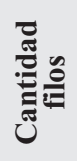 & 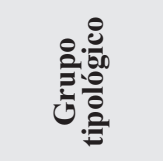 & שِّ & 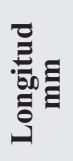 & 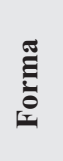 & 을 & $\stackrel{\mathscr{b}}{\mathscr{\rho}}$ & 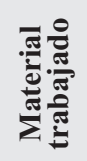 & : \\
\hline FCS.LB1.5150 & $\mathrm{E}$ & $\mathrm{C}$ & 1 & RAS & $\mathrm{MR} / \mathrm{RQ}-\mathrm{M}$ & 13 & $\mathrm{CV}$ & $38^{\circ}$ & US & MBA & $\mathrm{T}$ \\
\hline \multirow{2}{*}{ FCS.LB1.5158 } & \multirow{2}{*}{$\mathrm{F}$} & \multirow{2}{*}{$\mathrm{C}$} & \multirow{2}{*}{2} & \multirow{2}{*}{ RAE } & RQ-M & $\mathrm{n} / \mathrm{d}$ & $\mathrm{CV}$ & $56^{\circ}$ & US & MD & $\mathrm{L}$ \\
\hline & & & & & RQ-M & $\mathrm{n} / \mathrm{d}$ & $\mathrm{n} / \mathrm{d}$ & $57^{\circ}$ & UP & I & I \\
\hline \multirow{2}{*}{ FCS.LB1.5163 } & \multirow{2}{*}{$\mathrm{E}$} & \multirow{2}{*}{$\mathrm{C}$} & \multirow{2}{*}{3} & \multirow{2}{*}{$\begin{array}{c}\text { COMP } \\
(\mathrm{RAS}+\mathrm{RAE})\end{array}$} & $\begin{array}{c}\text { MR } \\
\text { MR/RQ-PE }\end{array}$ & $\begin{array}{c}24 \\
35,7\end{array}$ & $\begin{array}{c}\mathrm{R} \\
\mathrm{CV}\end{array}$ & $\begin{array}{l}40^{\circ} \\
54^{\circ}\end{array}$ & $\begin{array}{l}\text { US } \\
\text { US }\end{array}$ & $\begin{array}{c}\text { M } \\
\text { MD }\end{array}$ & $\begin{array}{l}\mathrm{T} \\
\mathrm{L}\end{array}$ \\
\hline & & & & & RQ-M & 15,6 & $\mathrm{CV}$ & $68^{\circ}$ & US & $\mathrm{P}$ & $\mathrm{T}$ \\
\hline FCS.LB1.5164 & $\mathrm{F}$ & FT & 1 & FBA & MR & $\mathrm{n} / \mathrm{d}$ & $\mathrm{CV}$ & $47^{\circ}$ & NDA & & \\
\hline FCS.LB1.5172 & $\mathrm{E}$ & $\mathrm{C}$ & 1 & RAE & RQ-M & 33,8 & $\mathrm{CV}$ & $59^{\circ}$ & NDA & & \\
\hline \multirow{2}{*}{ FCS.LB1.5175 } & $\mathrm{F}$ & $\mathrm{C}$ & 1 & FND & RQ-M & $\mathrm{n} / \mathrm{d}$ & $\mathrm{R}$ & $40^{\circ}$ & NU & & \\
\hline & \multirow{3}{*}{ E } & \multirow{3}{*}{ FT } & \multirow{3}{*}{3} & \multirow{3}{*}{ RAS } & RQ-M & 12,5 & $\mathrm{CV}$ & $55^{\circ}$ & NDA & & \\
\hline \multirow[t]{2}{*}{ FCS.LB1.5177 } & & & & & RQ-M & 13,5 & $\mathrm{CV}$ & $57^{\circ}$ & NDA & & \\
\hline & & & & & MR/RQ-M & 15,8 & $\mathrm{CV}$ & $65^{\circ}$ & US & $\mathrm{P}$ & $\mathrm{T}$ \\
\hline FCS.LB1.5190 & $\mathrm{F}$ & $\mathrm{C}$ & 1 & FND & RQ-M & $\mathrm{n} / \mathrm{d}$ & $\mathrm{R}$ & $40^{\circ}$ & UP & I & I \\
\hline & & & & & RQ-PE & 45,1 & $\mathrm{CV}$ & $64^{\circ}$ & US & $\mathrm{P}$ & $\mathrm{L}$ \\
\hline FCS.LB1.5192 & $\mathrm{E}$ & $\mathrm{C}$ & $2+1$ & RAE & RQ-PE & 45,1 & $\mathrm{CV}$ & $55^{\circ}$ & US & $\mathrm{P}$ & $\mathrm{L}$ \\
\hline & & & & & PUNT & $\mathrm{DEST}$ & ADA & & US & $\mathrm{P}$ & $\mathrm{R}$ \\
\hline FCS.LB1.5203 & $\mathrm{F}$ & $\mathrm{C}$ & 1 & MR & RQ-M & 20,1 & $\mathrm{CC}$ & $69^{\circ}$ & NU & & \\
\hline & & & & & RQ-M & $\mathrm{n} / \mathrm{d}$ & $\mathrm{n} / \mathrm{d}$ & $74^{\circ}$ & NDA & & \\
\hline FCS.LB1.5226 & $\mathrm{F}$ & $\mathrm{C}$ & 2 & FND & RQ-M & $\mathrm{n} / \mathrm{d}$ & $\mathrm{n} / \mathrm{d}$ & $50^{\circ}$ & US & MD & $\mathrm{L}$ \\
\hline FCS LB1 5231 & $\mathrm{E}$ & $\mathrm{C}$ & 2 & RAF & RQ-PE & 44,3 & $\mathrm{CV}$ & $49^{\circ}$ & UP & I & I \\
\hline FCS.LD & E & c & 2 & RAE & RQ-PE & 44,1 & $\mathrm{CV}$ & $54^{\circ}$ & US & $\mathrm{P}$ & $\mathrm{T}$ \\
\hline & & & & & MR & 33,2 & $\mathrm{CV}$ & $50^{\circ}$ & UP & MBA & I \\
\hline FCS.LB1.5241 & $\mathrm{E}$ & $\mathrm{C}$ & 3 & (RAS+RAE) & RQ-M & 38,7 & $\mathrm{CV}$ & $46^{\circ}$ & US & MD & $\mathrm{L}$ \\
\hline & & & & (KAS+KAE) & RQ-PE & 17 & $\mathrm{R}$ & $59^{\circ}$ & US & $\mathrm{P}$ & $\mathrm{T}$ \\
\hline FCS.LB1.5244 & $\mathrm{F}$ & $\mathrm{C}$ & 1 & RAE & MR/RQ-M & $\mathrm{n} / \mathrm{d}$ & $\mathrm{R}$ & $53^{\circ}$ & UP & MD & I \\
\hline & & & & & RQ-M & 13 & $\mathrm{CV}$ & $52^{\circ}$ & NDA & & \\
\hline FCS.LB1.5245 & $\mathrm{E}$ & $\mathrm{FT}$ & 2 & RAS & RQ-M & 12 & $\mathrm{CV}$ & $70^{\circ}$ & NDA & & \\
\hline FCS.LB1.5251 & $\mathrm{F}$ & $\mathrm{C}$ & 2 & & MR/RQ-M & 36,6 & $\mathrm{CV}$ & $56^{\circ}$ & US & M & $\mathrm{T}$ \\
\hline FCS.LB 1.5251 & $\mathrm{~F}$ & C & 2 & RAE & RQ-M & 27,7 & $\mathrm{CV}$ & $60^{\circ}$ & US & M & $\mathrm{T}$ \\
\hline FCS.LB1.5258 & $\mathrm{F}$ & $\mathrm{C}$ & 1 & FND & RQ-M & $\mathrm{n} / \mathrm{d}$ & $\mathrm{n} / \mathrm{d}$ & $\mathrm{n} / \mathrm{d}$ & NU & & \\
\hline FCS.LB1.5274 & $\mathrm{F}$ & $\mathrm{C}$ & 2 & RAE & RQ-M & 16,6 & $\mathrm{R}$ & $60^{\circ}$ & US & MD & $\mathrm{L}$ \\
\hline CES.LD 1.5214 & $\Gamma$ & C & 2 & KAE & RQ-M/RT-PE & 25,7 & $\mathrm{CV}$ & $48^{\circ}$ & UP & I & I \\
\hline & & $\mathrm{F}$ & 2 & RAS & RQ-E & 17,9 & $\mathrm{CV}$ & $73^{\circ}$ & NDA & & \\
\hline FCS.LB1.5342 & $\mathrm{E}$ & $\mathrm{F}$ & 2 & & MR/RQ-PE & 13,9 & $\mathrm{CV}$ & $63^{\circ}$ & NDA & & \\
\hline & & & & COMP & RQ-M & 19,7 & $\mathrm{CV}$ & $74^{\circ}$ & NDA & & \\
\hline FCS.LB1.56/1 & $\mathrm{F}$ & D & 2 & (RAS+FRS) & RQ-M & 6,9 & $\mathrm{CV}$ & $67^{\circ}$ & NDA & & \\
\hline & & & & & RQ-PE & $\mathrm{n} / \mathrm{d}$ & $\mathrm{CV}$ & $64^{\circ}$ & US & MBA & $\mathrm{L}$ \\
\hline FCS.LB 1.5672 & $\mathrm{~F}$ & $\mathrm{C}$ & 2 & RAE & RQ-M & $\mathrm{n} / \mathrm{d}$ & $\mathrm{CV}$ & $57^{\circ}$ & US & MBA & $\mathrm{T}$ \\
\hline FCS.LB1.5684 & & $\mathrm{C}$ & & & RQ-M & $\mathrm{n} / \mathrm{d}$ & $\mathrm{R}$ & $34^{\circ}$ & UP & I & I \\
\hline FCS.LBI.5684 & $\mathrm{F}$ & c & 2 & CFK & RQ-M & $\mathrm{n} / \mathrm{d}$ & $\mathrm{R}$ & $31^{\circ}$ & UP & I & I \\
\hline & & & & & RQ-M & 11 & $\mathrm{CV}$ & $56^{\circ}$ & NU & & \\
\hline FCS.LB1.5788 & $\mathrm{E}$ & D & 3 & RAS & RQ-PE & 14,1 & $\mathrm{CV}$ & $58^{\circ}$ & US & MBA & $\mathrm{T}$ \\
\hline & & & & & RQ-PE & 10,8 & $\mathrm{CV}$ & $65^{\circ}$ & NU & & \\
\hline FCS.LB1.6158 & E & $\mathrm{S}$ & 1 & RAS & RQ-M & 10,2 & $\mathrm{CV}$ & $73^{\circ}$ & NDA & & \\
\hline FCS.LB1.6255 & $\mathrm{E}$ & $\mathrm{C}$ & 1 & RAE & RQ-M & 40 & $\mathrm{CC}$ & $59^{\circ}$ & US & $\mathrm{H}$ & $\mathrm{T}$ \\
\hline
\end{tabular}

Clave: $\mathrm{F}=$ fracturado; $\mathrm{E}=$ entero; $\mathrm{C}=$ ortocuarcita $\mathrm{FT}=\mathrm{ftanita} ; \mathrm{D}=$ dolomía silicificada; $\mathrm{S}=$ roca silícea; $\mathrm{FND}=$ fragmento no diferenciado de instrumento; $\mathrm{RAE}=$ raedera; $\mathrm{COMP}=$ artefactos compuesto; $\mathrm{RAS}=$ raspador; $\mathrm{FRS}=$ filo con retoque sumario; $\mathrm{PE}=$ perforador; $\mathrm{FBA}=$ filo bisel asimétrico; $\mathrm{MU}=$ muesca retocada; $\mathrm{CFR}=$ cuchillo filo retocado; $\mathrm{MR}=$ microretoque; $\mathrm{RQ}=$ retoque; $\mathrm{RQ}-\mathrm{M}=$ retoque marginal; $\mathrm{RQ}-\mathrm{PE}=$ retoque parcialmente extendido; $\mathrm{RQ}-\mathrm{E}=$ retoque extendido; $\mathrm{RT}-\mathrm{PE}=$ retalla parcialmente extendida; $\mathrm{RT}-\mathrm{E}=$ retalla extendida; $\mathrm{NA}=$ no aplica por fractura; $\mathrm{CV}=$ convexo; $\mathrm{CC}=$ cóncavo; $\mathrm{R}=$ recto; $\mathrm{NDA}=$ no determinado por alteración; $\mathrm{US}=$ uso seguro; $\mathrm{UP}=$ uso posible; $\mathrm{NU}=$ no utilizado; $\mathrm{H}=$ hueso; $\mathrm{I}=$ indeterminado; $\mathrm{M}=$ madera; $\mathrm{MD}=\mathrm{material}$ duro; $\mathrm{P}=$ piel; $\mathrm{MBA}=$ material blando de origen animal; $\mathrm{L}=$ longitudinal; $\mathrm{T}=$ transversal; $\mathrm{R}=$ rotatorio. 
corresponden a los artefactos elaborados sobre las rocas caracterizadas como homogéneas (ftanita y dolomía), mientras que sólo se encuentran alterados dos filos sobre la roca clasificada como heterogénea (ortocuarcita) (ver definiciones de rocas homogéneas y heterogéneas en Mansur 1999). Con respecto a los filos que no presentan alteraciones severas, el análisis funcional posibilitó determinar que un $84,8 \%(\mathrm{n}=28)$ presenta huellas de uso y que un $15,2 \%(n=5)$ no fue utilizado.

En relación con los recursos procesados, se identificó el trabajo de piel $(25 \% ; n=7)$, materiales blandos de origen animal $(17,9 \% ; n=5)$, madera $(10,7 \% ; n=3)$ y hueso $(3,6 \% ; n=1)$. En algunos casos, sólo se pudo determinar que el material trabajado era duro, pudiendo corresponder a hueso o madera $(21,4 \% ; n=6)$, y en otros el material no pudo ser identificado dado el poco desarrollo de las huellas de uso $(21,4 \% ; n=6)$. Esta situación puede estar relacionada con el poco tiempo de uso de los artefactos o con el procesamiento de materiales blandos que llevan mayor tiempo en el desarrollo de huellas. Con respecto al movimiento, el más representado es el de tipo transversal $(39,3 \% ; n=11)$, seguido por el longitudinal $(28,6 \% ; n=8)$ y el rotatorio $(3,6 \% ; n=1)$. En el 28,6\% (n=8) de los filos no se logró reconocer el movimiento dado el escaso desarrollo de los micropulidos y la escasa presencia de estrías de uso.

Por otra parte, no se han identificado huellas de uso sobre un mismo filo producidos por el trabajo sobre distintos materiales y/o con distintos movimientos. No obstante, se ha observado el empleo de distintos filos de un mismo artefacto y, en algunos casos, estos se han usado para la ejecución de actividades diferentes (p.e., desbaste de madera y raspado de pieles). En este sentido, en un $60 \%(\mathrm{n}=9)$ de los artefactos con más de un filo, se identifican huellas de utilización en más de uno de ellos (36\% del total de piezas incluidas en el análisis funcional). Entre estos artefactos, se observa el uso de diferentes filos para trabajar el mismo material aunque con distinto movimiento, diferentes materiales con distintos movimientos y diferentes materiales con la misma cinemática (Cuadro 3).

Como se muestra en la Figura 4, los resultados del análisis funcional indican que los artefactos líticos de LB1 se emplearon principalmente en actividades que involu-

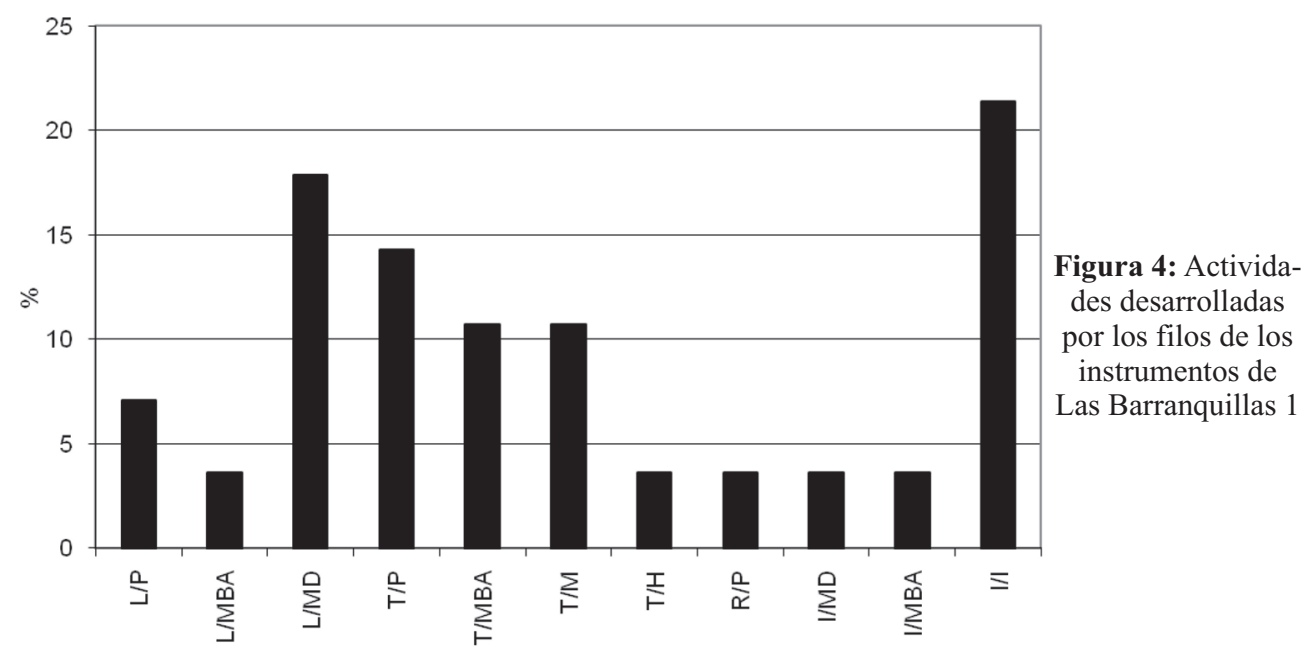


Figura 5: Filos en raedera utilizados en

Las Barranquillas 1.

FCS.LB1.5231:

raspado de pieles.

FCS.LB1.5251:

desbaste de madera
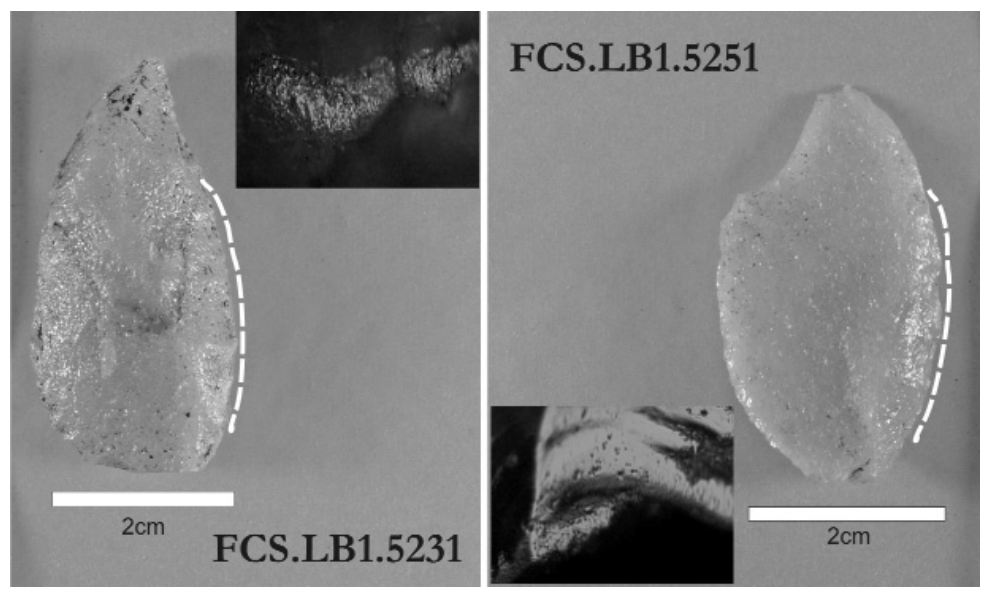

craron el corte de materiales duros no determinados, tales como hueso y/o madera $(17,9 \%)$, el raspado de pieles (14,3\%; Figura 5) y materiales blandos de origen animal $(10,7 \%)$ y el desbaste de madera (10,7\%; ver Figura 5). Además, aunque con menor frecuencia, se identificó su empleo en el corte y perforado de pieles $(7,1 \%$ y $3,6 \%$, respectivamente), corte de materiales blandos de origen animal $(3,6 \%)$ y raspado de hueso $(3,6 \%)$. Las huellas correspondientes al corte de materiales duros y al raspado sobre hueso podrían ser en parte el resultado del contacto de los artefactos líticos con los elementos óseos durante la realización de actividades de procesamiento de las presas. Por otra parte, las características y extensión de los micropulidos, conjuntamente con la diversidad de acciones desarrolladas sobre las pieles (raspado, corte, perforación), sugieren su procesamiento intensivo. Las propiedades de las huellas de uso desarrollados indican que las pieles se trabajaron tanto en estado fresco como seco y que, en algunos casos, se hizo uso de abrasivos, condiciones evidenciadas por la cantidad de estrías de uso observadas en los filos.

\subsection{Integración de la información tecno-morfológica y funcional}

Los instrumentos que presentan evidencias de utilización se encuentran manufacturados sobre ortocuarcita GSB $(n=18)$, ftanita $(n=4)$, dolomía $(n=2)$ y una roca silícea no determinada $(\mathrm{n}=1)$. Entre los filos utilizados, se registran 18 filos en raedera, 5 filos en raspador, 2 filos en cuchillo, 2 fragmentos de filos de artefactos no diferenciados y 1 punta destacada.

En cuanto a la relación entre las categorías tecno-morfológicas, material trabajado y cinemática (Cuadro 2), se observa que los filos en raedera fueron empleados para cortar y raspar pieles y materiales blandos de origen animal, desbastar madera, raspar hueso y aserrar materiales duros; los filos en raspador para raspar pieles y materiales blandos de origen animal; la punta retocada para perforar pieles y uno de los filos de los fragmentos de artefactos para el trabajo longitudinal sobre material duro no determinado. Es importante aclarar que se observaron diferencias en las funciones desarrolladas por algunos filos correspondientes a distintas categorías tecno-morfológicas que forman parte de los artefactos compuestos (ver Cuadro 3). 
Con respecto a la relación entre la serie técnica y forma del bisel y las actividades desempeñadas por los filos con evidencias de utilización en LB1 (ver Cuadro 3), se presenta la misma situación que en CO. De esta manera, no se observa una selección de filos de acuerdo con estas características para desarrollar una tarea específica. Por otra parte, el promedio del largo de los filos del sitio LB1 es de 29,6 mm y las principales diferencias se observan en las longitudes medias de los filos que rasparon material blando de origen animal y cuero $(20,2 \mathrm{~mm})$ y los que aserraron material duro $(30,3 \mathrm{~mm})$. Con referencia a los ángulos, la totalidad se incluye dentro de la categoría agudos. No obstante, no se registran diferencias que relacionen las actividades y los ángulos buscados, dado que hay una clara superposición entre los ángulos que procesaron diversos recursos y las acciones involucradas.

\section{Discusión}

La información resultante de la integración del análisis tecno-morfológico y funcional, tal como se ha aplicado al estudio de los conjuntos líticos de los sitios Cortaderas y Las Brusquillas 1, permite una mejor aproximación a las estrategias tecnológicas involucradas en la producción y uso de los instrumentos líticos que la que se efectúa desde sólo una línea de análisis. En los párrafos siguientes vamos a proceder a la discusión de los resultados obtenidos. Lo hacemos, en primer lugar, a escala de cada sitio, a fin de contribuir a la caracterización de su funcionalidad, y, en segundo lugar, a una escala espacial mayor, con el propósito de contribuir al conocimiento de las estrategias tecnológicas implementadas por los grupos cazadores-recolectores que habitaron el Área Interserrana durante el Holoceno tardío, para evaluar la relación entre diversidad tecno-morfológica y variabilidad funcional.

\subsection{Actividades desarrolladas en los sitios y funcionalidad}

Los estudios realizados en los sitios Cortaderas y Las Brusquillas 1, con anterioridad al análisis funcional, han permitido identificar parte de las actividades desarrolladas en ellos. En este sentido, se ha propuesto que el Componente Inferior del sitio CO es el resultado de ocupaciones de carácter residencial durante las cuales se llevaron a cabo tareas vinculadas a la manufactura de artefactos líticos y al procesamiento y consumo de una gran variedad de recursos animales. Por otro lado, se planteó también que durante las ocupaciones del sitio LB1 representadas en el Componente Inferior, los humanos procesaron y consumieron recursos animales y manufacturaron instrumentos líticos, aunque se sugirió que las actividades desarrolladas en este sitio fueron de carácter más específico que en CO. Con respecto a la funcionalidad de LB1 se propuso que podría corresponder a: 1) un sector de un campamento residencial en el cual se procesaron y consumieron algunos de los recursos animales cazados, o 2) un sitio de procesamiento secundario, en el cual se descarnaron algunas partes anatómicas para facilitar el transporte de las carcasas hacia el campamento residencial (Massigoge 2009).

En cuanto al sitio CO, los resultados del análisis de huellas de utilización sobre los instrumentos coinciden con la caracterización funcional previa y aportan nueva infor- 
mación que amplía el conocimiento de la gama de actividades desarrolladas. La realización de actividades de procesamiento en el sitio fue sugerida por la presencia de huellas de corte en especímenes óseos de guanaco (Lama guanicoe), venado de las pampas (Ozotoceros bezoarticus), vizcacha (Lagostomus maximus) y zorro (Dusicyon sp.). La identificación de estas marcas, así como el registro de otras modificaciones culturales sobre los huesos (p.e., alteración térmica, fracturas de tipo dinámico asociadas a la extracción de la médula y/o a la manufactura de artefactos óseos), llevó a plantear el consumo en términos alimenticios de los tres primeros taxones así como de armadillos (Dasipodidae), y el probable aprovechamiento de otros productos ofrecidos por estos recursos, como cuero, tendones y huesos, con propósitos tecnológicos. En cuanto a los zorros, se propuso el aprovechamiento de sus pieles, aunque no se pudo determinar su consumo como alimento (Massigoge 2009).

El procesamiento de animales en $\mathrm{CO}$ es apoyado por la alta frecuencia de huellas sobre los filos líticos correspondientes al trabajo sobre hueso, pieles y material blando de origen animal. Las evidencias de aserrado de hueso y, probablemente también, parte de las asignadas al corte de materiales duros, permiten proponer la participación del instrumental lítico en el trabajo del cuero, la desarticulación y/o el descarne de las carcasas. Por otra parte, las huellas de raspado y perforación de pieles, así como aquellas asignadas al raspado de material blando de origen animal, permiten plantear su utilización en el procesamiento intensivo de las pieles de las presas consumidas y, posiblemente, de los zorros. Por último, las evidencias funcionales del empleo del instrumental lítico en el aserrado de madera indican la realización de otro tipo de actividades no detectadas mediante otros tipos de análisis. En este sentido, se plantea que los filos líticos pudieron haber sido utilizados para la manufactura de instrumentos o piezas de madera, tales como puntas, mangos y/o astiles, raramente preservados en el registro arqueológico pampeano.

Del mismo modo, la información obtenida mediante el análisis funcional de los instrumentos de LB1 permite avanzar en la caracterización funcional de este sitio. De acuerdo con este estudio, una parte importante de las actividades desarrolladas en este sitio se relaciona con el procesamiento de los recursos animales. El claro predominio en el conjunto faunístico de especímenes de guanaco, así como el registro entre ellos de huellas de corte, marcas de raspado y fracturas de carácter dinámico, llevaron a plantear que en el sitio se procesaron fundamentalmente carcasas de este ungulado (Massigoge 2009). Coincidiendo con esta interpretación previa, se propone que algunos de los artefactos con huellas correspondientes al corte de materiales duros y al raspado sobre hueso pudieron haber sido utilizados durante las actividades de desarticulación y/o descarne de las carcasas.

Por otra parte, la consideración de distintas evidencias (presencia de pigmentos, abundancia de raspadores, fragmentos líticos que pudieron haber sido agregados como partículas abrasivas durante las tareas de sobado y un posible sobador), llevó a proponer que en LB1 también se llevó a cabo el procesamiento de los cueros de los animales cazados (Massigoge 2009). Los resultados del análisis funcional confirman esta interpretación y la amplían al demostrar que en el sitio se llevó a cabo el procesamiento intensivo de pieles en estado fresco y seco, incluyendo la realización de actividades de raspado, corte y perforación y que, en algunos casos, se recurrió al empleo de abra- 
sivos. Por último, el estudio funcional ha puesto en evidencia que en LB1 también se desarrollaron actividades vinculadas al procesamiento de recursos vegetales al igual que en el sitio CO. En este sentido, las huellas de uso que indican el desbaste de madera, sugieren que en este sitio se puede haber llevado a cabo la manufactura y/o el mantenimiento de dispositivos de enmangue de los instrumentos líticos u otros tipos de artefactos que no se conservaron (p.e., astiles, estacas).

Esta caracterización más detallada de las actividades realizadas en LB1 permite avanzar en la definición de la funcionalidad de este sitio, la cual no pudo ser precisada sobre la base de los estudios previos efectuados. La importancia del procesamiento de pieles en el sitio, tanto en estado fresco como seco, así como el trabajo que involucra la manufactura de instrumentos o piezas de madera, no concuerdan con la hipótesis que plantea que el CI de LB1 es el resultado de un sitio de procesamiento secundario. Consecuentemente, se propone que este componente sería el producto de ocupaciones de carácter residencial. No obstante, como ha sido previamente mencionado, hay un claro contraste entre este sitio y $\mathrm{CO}$, el cual también fue caracterizado como un campamento residencial. Considerando los resultados del análisis faunístico y lítico, se puede proponer que LB1 corresponde a un campamento residencial en el cual una de las actividades centrales fue el procesamiento de pieles.

En suma, el análisis funcional de base microscópica permitió obtener un conocimiento más preciso de las actividades desarrolladas en CO y LB1, contribuyendo a la identificación del contexto de uso de los instrumentos líticos. Por otra parte, las diferencias en las huellas de uso identificados en cada uno de los conjuntos de artefactos por grupo tipológico son de suma utilidad para discutir en los párrafos subsiguientes la relación entre variabilidad tipológica y funcional.

\subsection{Integración regional de la información tecno-morfológica y funcional}

Si se comparan los resultados obtenidos en los sitios CO y LB1, se identifican ciertas similitudes en las estrategias tecnológicas implementadas. En este sentido, la roca más seleccionada para la manufactura de instrumentos en ambos conjuntos es la ortocuarcita GSB de carácter no local. El amplio predominio de esta roca en los sitios de la micro-región coincide con lo observado en la mayoría de los sitios del Holoceno tardío del Área Interserrana (Bayón et al. 2006; Crivelli Montero et al. 1997; Martínez y Mackie 2003; Politis 1986). Su elección preferencial puede obedecer en parte a que esta roca constituye una de las materias primas de mejor calidad para la talla disponible en la sub-región Pampa Húmeda (Bayón et al. 1999). Asimismo, desde el punto de vista funcional, Leipus y Mansur (2007) plantean que la ortocuarcita GSB pudo ser seleccionada por las sociedades cazadoras-recolectoras del Área Interserrana porque permite obtener lascas medianas y grandes para confeccionar filos largos y resistentes a diversos procesos de uso. El presente estudio apoya esta idea, ya que se observa en ambos conjuntos analizados el empleo de la ortocuarcita para la manufactura de instrumentos sobre lascas de estos tamaños, con filos mayormente largos, los cuales fueron utilizados para el desempeño de múltiples actividades.

La ausencia de rocas aptas para la talla en la micro-región de estudio requirió del transporte, por parte de las poblaciones, de materia prima y/o artefactos hacia los lu- 
gares en donde se explotaban los recursos de subsistencia. El abastecimiento de las principales rocas utilizadas en la micro-región se realizó probablemente en el sistema serrano de Tandilia, a unos $80 \mathrm{~km}$ de distancia de los sitios estudiados, donde se han localizado distintos complejos de cantera-taller de estas materias primas (Flegenheimer et al. 1996). Las rocas fueron transportadas en forma de núcleos, formas base e instrumentos, y habrían llegado a los sitios de la micro-región en un avanzado estado de reducción. En los conjuntos predomina una tecnología orientada a la producción de lascas, las cuales se seleccionaban como soporte para la manufactura de los instrumentos, confeccionados mayoritariamente por retoque marginal en situación unifacial directa. Como mencionamos anteriormente, los instrumentos confeccionados sobre ortocuarcita fueron utilizados en una gran variedad de actividades, coincidiendo con lo observado en otros sitios del Área Interserrana (Castro de Aguilar 1987-1988; Leipus 2006; Leipus y Mansur 2007; Pal 2009b). Con respecto a los instrumentos de otras materias primas, el reducido tamaño de la muestra y la alta frecuencia de filos alterados no permiten evaluar si fueron destinados a usos diversos o específicos.

En relación con los tipos de instrumentos utilizados para efectuar las distintas actividades, los resultados obtenidos permiten plantear que en general no existe una tendencia hacia el uso de grupos tipológicos particulares para el desarrollo de funciones específicas. En este sentido, se propone que las raederas y los artefactos compuestos fueron instrumentos multifuncionales, empleados en el procesamiento de distintos recursos y con diferentes cinemáticas. La excepción son los raspadores, los cuales se vincularon principalmente con el trabajo de las pieles, siendo este grupo tipológico el único que presenta mayor integridad funcional. La baja cantidad de otros grupos tipológicos en las muestras no permitió evaluar si fueron destinados a usos particulares. Estos resultados coinciden con aquellos obtenidos por diversos autores para otros sitios del Área Interserrana, en los cuales en general observan el uso de un mismo grupo tipológico para el desarrollo de distintas actividades (p.e., raederas, filos en bisel asimétrico, etc.), con la excepción de los raspadores, usados en su totalidad para el trabajo transversal y, mayoritariamente, para el procesamiento de pieles (Landini et al. 2000; Leipus 2006; Leipus y Mansur 2007).

Con respecto a los filos, no se han identificado relaciones claras entre algunas de sus características (forma y serie técnica) y las actividades que se llevaron a cabo. Las únicas diferencias observadas en los datos analizados son con respecto a la longitud y al ángulo del filo. En este sentido, se puede formular la elección preferencial de filos largos para trabajos de corte y/o aserrado de madera y/o materiales duros y más cortos para acciones de raspado de pieles, ya que estos últimos permiten una mayor concentración de la fuerza. Asimismo, si bien se observa en uno de los sitios una tendencia hacia la utilización de los filos más agudos para las actividades de corte de madera, se registra en general una superposición en los ángulos utilizados para el desarrollo de distintas cinemáticas.

Como ya se ha planteado para otros sitios del Área Interserrana, el empleo de instrumentos multifuncionales, así como de filos generalizados por parte de las sociedades prehistóricas, podría vincularse a la importancia de la versatilidad en el diseño (Leipus y Mansur 2007; Pal 2009b). En este sentido, puede proponerse que las similitudes observadas a nivel tecno-morfológico en los dos conjuntos analizados no obe- 
dece necesariamente a semejanzas en las actividades realizadas en ellos, sino al empleo de instrumentos multifuncionales adecuados para el desarrollo de una amplia diversidad de tareas. El empleo de conjuntos de artefactos diseñados de modo versátil es una estrategia tecnológica adecuada para grupos con una alta movilidad residencial en un ambiente carente de materias primas (tal como el área de estudio), debido a que, con un reducido costo de transporte del instrumental, permite contar con un amplio rango de opciones de uso (Nelson 1991; Shott 1986).

Por otra parte, la alta frecuencia de instrumentos con filos dobles o múltiples sugiere un gran aprovechamiento de los bordes de las lascas para la confección de filos, lo cual podría relacionarse con un uso intensivo del recurso lítico (Jeske 1989; Kuhn 1995). La hipótesis de aprovechamiento intensivo de las materias primas por parte de los grupos cazadores-recolectores que ocuparon la micro-región fue propuesta previamente sobre la base de la alta frecuencia de núcleos bipolares agotados de ortocuarcita y ftanita y el hallazgo de algunos desechos bipolares del primer tipo de roca (Massigoge 2009).

Considerando una serie de características de los conjuntos líticos del área de estudio, incluyendo el avanzado estado de reducción de los artefactos, la baja frecuencia de núcleos y su pequeño tamaño y la versatilidad en el diseño de los instrumentos, se propone que son el resultado del aprovisionamiento de los individuos (Kuhn 1995). Por el momento no se ha identificado en la micro-región la implementación de una estrategia de aprovisionamiento de los lugares vinculada a un uso programado de determinados espacios, la cual ha sido reconocida en sectores aledaños del Área Interserrana, entre ellos, la cuenca media del río Quequén Grande y la llanura adyacente al litoral atlántico (ver Bayón y Flegenheimer 2004; Bonomo 2005; Martínez y Mackie 2003). Las discrepancias entre el registro de la micro-región con el de sectores vecinos pueden deberse a diferencias en la dinámica de ocupación del espacio regional. En este sentido, se plantea que la mayor continuidad en la disponibilidad de agua y recursos asociados en las cuencas mayores, como el río Quequén Grande al noreste de la micro-región, o en la porción de llanura colindante con el litoral atlántico, habría favorecido su ocupación más intensa, una menor movilidad residencial y el uso programado de los espacios. En el área de estudio, el menor desarrollo de las cuencas fluviales sugiere la existencia de mayores fluctuaciones estacionales o interanuales en la disponibilidad de agua, que podrían haber influido en la dinámica de las ocupaciones humanas. De esta manera, se plantea una mayor movilidad residencial de los grupos dentro de la micro-región con respecto a los sectores arriba mencionados, manifestándose en el carácter temporal de las ocupaciones y en la ausencia de indicadores de uso anticipado de estos espacios. El área de estudio habría formado parte del territorio de explotación de los mismos grupos que ocupaban los sectores adyacentes de la llanura Interserrana, los sistemas serranos, y el litoral atlántico, aunque el establecimiento de campamentos residenciales dentro de ella se habría visto más afectado por las variaciones en la disponibilidad de agua (Massigoge 2009).

En este contexto, se puede plantear para el área de estudio durante el Holoceno tardío, la implementación de estrategias de aprovisionamiento de los individuos con conjuntos de artefactos transportables y versátiles. Estos equipos les habrían permitido a los grupos cazadores-recolectores desarrollar las diversas tareas necesarias para la re- 
producción económica y social. Debido a la ausencia de rocas dentro de la micro-región, el abastecimiento de las materias primas líticas para la manufactura de estos conjuntos debió haberse realizado en los distintos sectores de la región pampeana, en donde las rocas aptas para la talla se encuentran naturalmente disponibles (particularmente en Tandilia) o en aquellos espacios cercanos en donde el recurso lítico se acumuló previamente como resultado del aprovisionamiento de los lugares. El aprovechamiento intensivo de los recursos líticos observado en los sitios de la microregión, podría ser consecuencia entonces, entre otros factores, del alto costo de transporte de la materia prima y de la alta movilidad de los grupos (Jeske 1989; Kuhn 1995; Nelson 1991; entre otros).

\section{Conclusiones}

En nuestros casos de estudio, el análisis de las huellas de uso ha posibilitado corroborar la funcionalidad adjudicada a $\mathrm{CO}$ a partir de otras líneas de evidencia así como dirimir entre las diversas funciones que habían sido propuestas para LB1. Asimismo, los datos alcanzados han permitido identificar el aprovechamiento de madera, material que generalmente no se preserva en la región pampeana, pero que a partir de datos etnográficos e históricos (Claraz [1865-1866] 1988; Musters 2005), se reconoce como imprescindible en el modo de vida cazador-recolector. Por otra parte, el análisis funcional ha corroborado ideas previas acerca del aprovechamiento intensivo de las materias primas en la micro-región y ha aportado nueva información acerca de la importancia de la versatilidad en el diseño de los conjuntos de artefactos que los individuos trasladaban con ellos para el desenvolvimiento de las distintas actividades necesarias para la reproducción económica y social.

Si comparamos sólo las muestras de instrumentos con huellas de uso de ambos sitios, no se observan contrastes en las características tecnológicas y en la composición tipológica. No obstante, se presentan diferencias a nivel de las actividades en las cuales los instrumentos participaron como herramientas de trabajo. En este sentido, si bien los filos revelan un amplio rango de funciones desarrolladas, las labores relacionadas con el procesamiento de cuero, madera y hueso no se desarrollaron con igual intensidad en ambos sitios. De este resultado se desprende que el estudio de la composición tipológica por sí solo puede enmascarar las actividades llevadas a cabo en los sitios estudiados. De esta manera, se debe tener en cuenta el hecho de no tomar exclusivamente la diversidad tipológica como criterio diagnóstico para definir la funcionalidad de los sitios.

Con el advenimiento de la metodología de análisis funcional de base microscópica se ha comenzado a discutir y replantear la vinculación entre la forma y función de los instrumentos líticos en el marco de la tecnología de cazadores-recolectores. Esta problemática es esencial para el análisis de la composición de los conjuntos de artefactos y la definición de la funcionalidad de los sitios, dado que las funciones adjudicadas a los diferentes grupos tipológicos, en algunos casos, han sido asumidas a priori a partir de analogías intuitivas entre conjuntos etnoarqueológicos y etnográficos, de un lado, y el registro arqueológico, de otro, o a partir de la identificación de macro-huellas (Civalero de Biset 1988; Franco y Carballo Marina 1993; Gould 1973; Yacobaccio 1987). 
A partir de los resultados obtenidos del análisis microscópico de las huellas de uso se explicita la importancia de no considerar la vinculación entre forma y función de manera unívoca. Nuestro estudio agrega nuevas evidencias de que los diversos grupos tipológicos no siempre se corresponden con los usos a los cuales han sido destinados y que instrumentos que poseen morfologías similares pueden haber tenido funciones distintas (ver conclusiones similares en: Álvarez 2003; Leipus 2006). Como ya se ha explicitado en este trabajo, el único instrumento que presenta integridad funcional es el de los raspadores, por lo cual, la inexistencia de una clara relación entre forma-función hace imposible explicitar los procesos productivos en los cuales participaron los instrumentos únicamente a través del análisis tecno-morfológico.

Agradecimientos: Este trabajo fue realizado en el marco de los proyectos doctorales de las autoras, llevados a cabo con el apoyo de CONICET y gracias al INCUAPA (FACSO, UNCPBA) y al CADIC (CONICET) que nos brindan un lugar de trabajo y la infraestructura necesaria para desarrollar nuestras investigaciones. Las autoras agradecen a Pablo Messineo, Myrian Álvarez y Patricia Madrid por la lectura crítica del manuscrito y los comentarios brindados. No obstante, las ideas vertidas en este trabajo son de exclusiva responsabilidad de las autoras.

\section{Referencias bibliográficas}

Álvarez, Myrian R.

2003 Organización tecnológica en el canal de Beagle. El caso de Túnel 1 (Tierra del Fuego, Argentina). Tesis doctoral inédita. Universidad de Buenos Aires.

2004 «¿A qué responde la diversidad instrumental? Algunas reflexiones a partir del análisis funcional de materiales líticos de la costa norte del canal de Beagle», en Contra viento y marea. Arqueología de Patagonia, María Teresa Civalero, Pablo M. Fernández y Ana G. Guráieb, comps. pp. 29-43. Buenos Aires: Instituto Nacional de Antropología y Pensamiento Latinoamericano - Sociedad Argentina de Antropología.

Aschero, Carlos

1975 «Ensayo para una clasificación morfológica de artefactos líticos aplicada a estudios tipológicos comparativos». Informe inédito presentado al CONICET, Buenos Aires.

1983 «Ensayo para una clasificación morfológica de artefactos líticos. Apéndice A y B». Apuntes inéditos de la cátedra de Ergología y Tecnología, Facultad de Filosofía y Letras, Universidad de Buenos Aires.

BAYÓn, Cristina y Nora FlegenneImer

2004 «Cambio de planes a través del tiempo para el traslado de roca en la pampa bonaerense». Estudios Atacameños 28: 59-70.

Bayón, Cristina, Nora Flegennheimer y Alejandra PuPIO

2006 «Planes sociales en el abastecimiento y traslado de roca en la pampa bonaerense en el Holoceno temprano y tardío». Relaciones de la Sociedad Argentina de Antropología 31: 19-45.

Bayón, Cristina, Nora Flegenheimer, Miguel Valente y Alejandra Pupio

1999 «Dime cómo eres y te diré de dónde vienes: procedencia de rocas cuarcíticas en la 
A. Massigoge y N. Pal Producción y uso de artefactos líticos en contextos cazadores-recolectores...

Región Pampeana». Relaciones de la Sociedad Argentina de Antropología 24: 187217.

BonOMO, Mariano

2005 Costeando las llanuras: Arqueología del litoral marítimo pampeano. Buenos Aires: Sociedad Argentina de Antropología.

Castro de Aguilar, Alicia

1987-88 «Análisis microscópico de huellas de utilización en artefactos líticos de Fortín Necochea». Paleoetnológicas 4: 65-78.

Civalero De Biset, María Teresa

1988 «Análisis morfológico-funcional de raspadores de los niveles de ocupación del cerro Casa de Piedra 5, Provincia de Santa Cruz». Trabajo presentado en el IX Congreso Nacional de Arqueología Argentina. Universidad de Buenos Aires, Buenos Aires.

ClaRAZ, Jorge

1988 Diario de viaje de exploración al Chubut. 1865-1866. Buenos Aires: Marymar.

Crivelli Montero, Eduardo A., Emilio Eugenio, Ulises F. Pardiñas y Mario J. Silveira

1997 «Archaeological Investigation in the Plains of the Province of Buenos Aires, Llanura Interserrana Bonaerense». Quaternary of South America and Antarctic Peninsula 10 (1994): 167-207.

FlegenheIMER, Nora y Cristina BAYÓN

2002 «Cómo, cuándo y dónde? Estrategias de abastecimiento lítico en la Pampa Bonaerense», en Del Mar a los Salitrales. Diez Mil Años de Historia Pampeana en el Umbral del Tercer Milenio, Diana L. Mazzanti, Mónica A. Berón y Fernando W. Oliva, eds., pp. 231-241. Mar del Plata: Facultad de Humanidades, UNMdP - Sociedad Argentina de Antropología.

FLEGENHEIMER, Nora y Marcela LEIPUS

2007 «Trabajar en un espacio reducido, Cerro El Sombrero, Abrigo 1», en Resúmenes Ampliados del XVI Congreso Nacional de Arqueología Argentina, tomo I, pp. 441444. San Salvador de Jujuy: Facultad de Humanidades y Ciencias Sociales, UNJu.

FlegenheIMER, Nora, Sergio KAIn, Marcelo ZÁrATE y Alejandra BARNA

1996 «Aprovisionamiento de cuarcitas en Tandilia, las canteras del Arroyo Diamante». Arqueología 6: 117-141.

Franco, Nora y Flavia CARBALlo Marina

1993 «Variabilidad en raederas en el lago Argentino (Santa Cruz, Argentina)». Arqueología 3: 213-232.

GoulD, Richard

1973 «Use Wear on Western Desert Aborigines Stone Tools: A Reply to Messrs, Hayden y Kamminga». Newsletter of Lithic Technology 2: 9-13.

JESKE, Robert

1989 «Economies in Raw Material Use by Prehistoric Hunter-Gatherer», en Time, Energy and Stone Tools, Robin Torrence, ed., pp. 34-45. Cambridge: Cambridge University Press.

KEELEY, Lawrence

1980 Experimental Determination of Stone Tool Uses. A Microwear Analysis. Chicago: The University of Chicago Press. 
KUHN, Steven L.

1995 Mousterian Lithic Technology. An Ecological Perspective. Princenton: Pricenton University Press.

LANDini, Cecilia, Mariano Bonomo, Marcela LeIPus y Gustavo MarTíneZ

2000 «Forma y función de los instrumentos líticos del sitio Paso Otero 3 (pdo. de Necochea, pcia. de Buenos Aires, Argentina): un estudio comparativo». Espacio, Tiempo y Forma, Serie I, Prehistoria y Arqueología 13: 161-187.

LEIPUS, Marcela

2006 Análisis de los modos de uso prehispánicos de las materias primas líticas en el sudeste de la región Pampeana. Tesis doctoral inédita. Universidad Nacional de La Plata.

LeIPus, Marcela y María Estela MANsur

2007 «El análisis funcional de base microscópica aplicado a materiales heterogéneos. Perspectivas metodológicas para el estudio de las cuarcitas de la Región Pampeana», en Arqueología en las Pampas, Cristina Bayón et al., eds., pp. 179-200. Buenos Aires: Sociedad Argentina de Antropología.

MANSUR, María Estela

1986-90 «Instrumentos líticos: Aspectos da analise funcional». Arquivos do Museu de Historia Natural 11: 115-169.

1999 «Análisis funcional de instrumental lítico: Problemas de formación y deformación de rastros de uso», en Actas del XII Congreso Nacional de Arqueología Argentina, tomo I, pp. 355-366. La Plata: Facultad de Ciencias Naturales y Museo, Universidad Nacional de La Plata.

MartíneZ, Gustavo A. y Quentin MackIE

2003 «Late Holocene Human Occupation of the Quequén Grande River Valley Bottom: Settlement Systems and an Example of a Built Environment in the Argentine Pampas». Before Farming [versión impresa] 2003 (4): 178-202.

Massigoge, Agustina

2007 «Resultados preliminares de las investigaciones arqueológicas desarrolladas en el partido de San Cayetano (provincia de Buenos Aires)», en Arqueología en las Pampas, Cristina Bayón et al., eds., pp. 511-534. Buenos Aires: Sociedad Argentina de Antropología.

2009 Arqueología de los cazadores-recolectores del sudeste de la región pampeana: una perspectiva tafonómica. Tesis Doctoral inédita. Universidad Nacional de La Plata.

Musters, George. C.

2005 Vida entre los patagones. Buenos Aires: El Elefante Blanco.

NELSON, Margaret C.

1991 «The Study of Technological Organization», en Archaeological Method and Theory 3, Michael B. Schiffer, ed., pp. 57-100. Tucson: The University of Arizona Press.

PAL, Nélida

2009a «Aproximaciones a las estrategias de uso e historias tafonómicas de los conjuntos líticos de los sitios ubicados en la cuenca superior del arroyo Tapalqué (Pdos. de Olavarría y Benito Juárez, Pcia. de Buenos Aires, Argentina)». Werken 12. [En prensa.] 
2009b «Correlación entre forma-función de los conjuntos líticos recuperados en la cuenca superior del arroyo Tapalqué (Pcia. de Buenos Aires): Su vinculación con otros sitios de la región pampeana», en Mamül Mapu: pasado y presente. Perspectivas de la arqueología pampeana a comienzos del tercer milenio, Mónica A. Berón et al., eds., pp. 309-322. Ayacucho: Libros del Espinillo.

Politis, Gustavo G.

1986 «Investigaciones arqueológicas en el Área Interserrana Bonaerense». Etnia 32: 752 .

Politis, Gustavo G., Eduardo TonNi y Francisco Fidalgo

1983 «Cambios corológicos de algunos mamíferos en el Área Interserrana de la Provincia de Buenos Aires durante el Holoceno». Ameghiniana 20 (1-2): 72-80.

SACUR SiLvestre, Romina

2004 «Análisis de rastros de uso en lascas de filo natural del sitio arqueológico Anahí», en Aproximaciones Contemporáneas a la Arqueología Pampeana. Perspectivas Teóricas, Metodológicas, Analíticas y Casos de Estudio, Gustavo Martínez et al., eds. pp. 183-201. Olavarría: Facultad de Ciencias Sociales, Universidad Nacional del Centro de la Provincia de Buenos Aires.

SHOTT, Michael

1986 «Technological Organization and Settlement Mobility: An Ethnographic Examination». Journal of Anthropological Research 42 (1): 15-51.

SEMENOV, Sergei A.

1964 Prehistoric Technology. Londres: Cory, Adams \& Mackay.

YACOBACCIO, Hugo

1987 «Los raspadores de Patagonia: un problema multifunción», en Actas de las Primeras Jornadas de Arqueología de la Patagonia, pp. 311-320. Rawson.

ZÁRATE, Marcelo y Jorge O. RABASSA

2005 «Geomorfología de la provincia de Buenos Aires», en Relatorio del XVI Congreso Geológico Argentino, Raúl E. de Barrio et al., eds., pp. 119-138. La Plata: Ministerio de la Producción Gobierno de la Provincia de Buenos Aires/Asociación Geológica Argentina. 